\title{
Results for light pseudoscalars from three-flavor simulations
}

\author{
The MILC Collaboration: C. Aubin,,${ }^{\mathrm{a}}$ C. Bernard, ${ }^{\mathrm{a}}{ }^{*}$ C. DeTar, ${ }^{\mathrm{b}}$ Steven Gottlieb, ${ }^{\mathrm{c}}$ E.B. Gregory, \\ Urs M. Heller, J.E. Hetrick, J. Osborn, ${ }^{\mathrm{b}}$ R.L. Sugarg ${ }^{\mathrm{f}}$ and D. Toussaint ${ }^{\mathrm{d}}$ \\ ${ }^{a}$ Department of Physics, Washington University, St. Louis, MO 63130, USA \\ ${ }^{b}$ Physics Department, University of Utah, Salt Lake City, UT 84112, USA \\ ${ }^{c}$ Department of Physics, Indiana University, Bloomington, IN 47405, USA \\ ${ }^{\mathrm{d}}$ Department of Physics, University of Arizona, Tucson, AZ 85721, USA \\ eAmerican Physical Society, One Research Road, Box 9000, Ridge, NY 11961, USA \\ ${ }^{f}$ Physics Department, University of the Pacific, Stockton, CA 95211, USA \\ g Department of Physics, University of California, Santa Barbara, CA 93106, USA
}

\begin{abstract}
We compute pseudoscalar meson masses and decay constants in partially quenched QCD with three dynamical flavors of improved staggered quarks. Fitting the lattice data to staggered chiral perturbation theory (S $\chi \mathrm{PT})$ forms and extrapolating in quark mass and lattice spacing, we find: $f_{\pi}=129.5 \pm 0.9 \pm 3.5 \mathrm{MeV}, f_{K}=156.6 \pm 1.0 \pm$ $3.6 \mathrm{MeV}$, and $f_{K} / f_{\pi}=1.210(4)(13)$. The value for $f_{K} / f_{\pi}$ implies $\left|V_{u s}\right|=0.2219(26)$. We also obtain $m_{u} / m_{d}=$ $0.43(0)(1)(8)$, or, with more input on electromagnetic (EM) effects from the continuum, $m_{u} / m_{d}=0.41(0)(1)(4)$. Using the one-loop perturbative mass renormalization [1, we determine $m_{u}$ and $m_{d}$ in the $\overline{\mathrm{MS}}$ scheme.
\end{abstract}

The properties of the $\pi$ - $K$ system are amenable to high-precision lattice computation. At fixed lattice spacing and quark mass, our improved staggered 2] computation achieves a statistical accuracy of $0.1 \%$ to $0.4 \%$ for decay constants and $0.1 \%$ to $0.7 \%$ for squared meson masses, down to $m_{\pi} \approx 250 \mathrm{MeV}\left(m_{\pi} / m_{\rho} \approx 0.3\right)$. This allows us to check that the chiral behavior is as expected from continuum chiral perturbation theory $(\chi \mathrm{PT})$ plus the controlled $\mathcal{O}\left(a^{2}\right)$ corrections predicted by $\mathrm{S} \chi \mathrm{PT}[3$. The check is especially important because our implementation of staggered fermions uses the as yet unproven $\sqrt[4]{\text { Det }}$ trick to move from 4 "tastes" (doublers) to 1 . With $\mathrm{S} \chi \mathrm{PT}$, the chiral and continuum extrapolations can then be performed to extract $f_{\pi}$ and $f_{K}$, quark masses and ratios, and several low energy constants. A detailed account of our methods and results appears in Ref. 4. Here, we give an overview and update our light quark mass results to take into account continuum analyses of EM effects.

Our computation uses seven lattice runs; five

*presented by C. Bernard at Lattice 2004, Fermilab, June 21-26, 2004 make up the "coarse" set $(a \approx 0.125 \mathrm{fm})$, with degenerate $u, d$ sea quark mass $a \hat{m}^{\prime}=0.005$ to 0.03 and strange sea quark mass $a m_{s}^{\prime}=0.05$, and two make up the "fine" set $(a \approx 0.09 \mathrm{fm})$, with $a \hat{m}^{\prime}=0.0062,0.0124$, and $a m_{s}^{\prime}=0.031$. (The primes distinguish simulation masses from their physical counterparts.) For Goldstone meson masses and decay constants we have extensive partially quenched data: all combinations of 8 or 9 valence masses between $0.1 m_{s}^{\prime}\left(0.14 m_{s}^{\prime}\right.$ on the fine set) and $m_{s}^{\prime}$. For other tastes, we have most of the full QCD pion masses but no decay constants and no partially quenched data.

Given the available data, we can only hope to make precise computations for Goldstone mesons. However, since mesons with other tastes affect the Goldstone properties through chiral loops, we need to know the masses of those mesons to reasonable precision. We get the needed splittings and the slope in quark mass from a treelevel S $\chi$ PT fit to full QCD data for pions of all tastes. Using these tree-level masses in the oneloop terms gives an NNLO error in the final results; since the maximum error in the tree-level fit 
is only $\approx 7 \%$, the error in the final results turns out to be well under $1 \%$, i.e., negligible.

To get good fits to $\mathrm{S} \chi \mathrm{PT}$ forms, we need to place upper limits on the valence quark masses $\left(m_{x}, m_{y}\right)$ considered. We consider 3 data subsets: subset $I$ (94 data points) with $m_{x}+m_{y} \leq$ $0.40 m_{s}^{\prime}$ (coarse) and $\leq 0.54 m_{s}^{\prime}$ (fine); subset $I I$ (240 points) with $m_{x}+m_{y} \leq 0.70 m_{s}^{\prime}$ (coarse) and $\leq 0.80 m_{s}^{\prime}$ (fine); and subset III (416 points) with $m_{x}+m_{y} \leq 1.10 m_{s}^{\prime}$ (coarse) and $\leq 1.14 m_{s}^{\prime}$ (fine). We can tolerate heavier valence masses (compared to $m_{s}^{\prime}$ ) on the fine lattices, since both $m_{s}^{\prime} / m_{s}$ and the taste splittings are smaller.

On subset $I$, the maximum valence-valence Goldstone mass is $\approx 350 \mathrm{MeV}$. Adding on the average taste splitting gives $\approx 500 \mathrm{MeV}$; the worst case is $\approx 580 \mathrm{MeV}$. Defining a chiral expansion parameter $\chi \equiv(500 \mathrm{MeV})^{2} /\left(8 \pi^{2} f_{\pi}^{2}\right)$, we thus expect the errors of NLO S $\chi \mathrm{PT}$ to be of order $\chi^{2} \approx 3.5 \%$. Since this is much larger than our statistical errors, NNLO terms are clearly needed, even for this lightest mass set. Unfortunately, the NNLO $\mathrm{S} \chi \mathrm{PT}$ logs have not been calculated. But for the higher masses where the NNLO terms are important, the logs should be smoothly varying and well approximated by NNLO analytic terms. Our "NNLO" fit functions therefore include the full NLO expressions supplemented by the analytic terms at NNLO. We fit decay constants and masses together, including all correlations; this constrains the new $\mathrm{S} \chi \mathrm{PT}$ hairpin parameters, which are common to both. We also fit coarse and fine lattices together, which constrains the overall lattice spacing dependence.

The NNLO fit has 20 unconstrained parameters: 2 at LO, 4 physical ones $\left(L_{i}\right)$ at NLO, 4 taste-violating ones at NLO, and 10 physical NNLO analytic parameters. There are an additional 16 tightly-constrained parameters that allow for the variation of physical parameters with $a\left(\sim \alpha_{S} a^{2} \Lambda_{\mathrm{QCD}}^{2} \approx 2 \%\right)$. Finally, 4 tightlyconstrained parameters allow the scale determinations to vary within statistical errors.

We are able to find good NNLO fits for subsets $I$ and $I I$. In subset $I I I$, even NNLO fits break down. Yet it is subset III that can allow us to interpolate around $m_{s}$. The strategy for subset $I I I$ is to fix the LO and NLO terms from lower mass

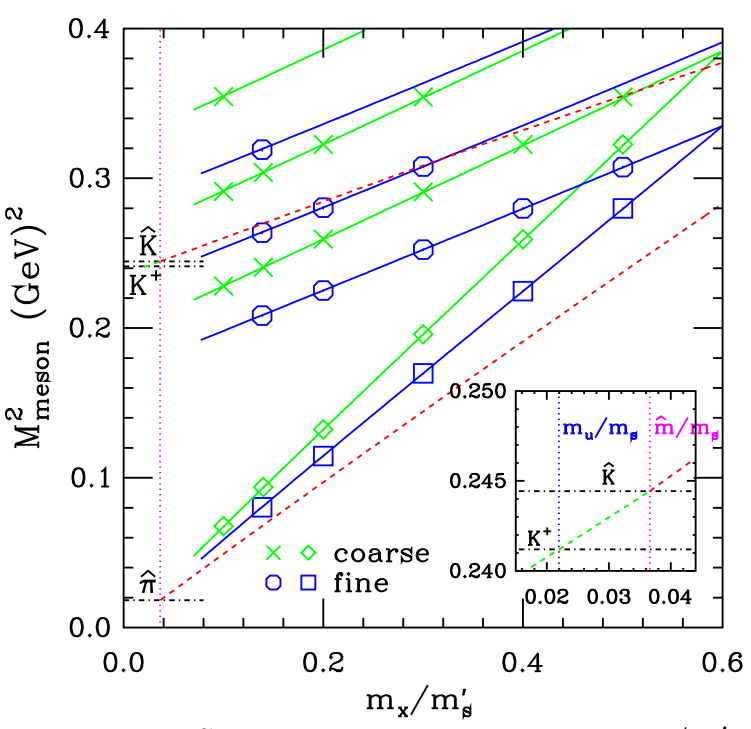

Figure 1. Squared meson masses vs. $m_{x} / m_{s}^{\prime}$. "Kaon" points (crosses and octagons) have $m_{y}=$ $m_{s}^{\prime}, 0.8 m_{s}^{\prime}$, or $0.6 m_{s}^{\prime}$; "pion" points (diamonds and squares) have $m_{x}=m_{y}$.

fits; then add on ad hoc higher order terms to get a good interpolating function around $m_{s}$. We use such fits for central values of quark masses and decay constants; results from the other subsets are included in systematic error estimates. Figure 1 shows the fit results in subset III for partiallyquenched meson masses; while Fig. [2] shows the same fit for pion decay constants. Points and fit lines in these plots have been corrected for finite volume effects at one-loop in $\mathrm{S} \chi \mathrm{PT}$.

To find physical values of the (bare) quark masses, we first extrapolate the chiral parameters to the continuum. The extrapolation is linear in the leading discretization errors, either $\alpha_{S}^{2} a^{2}$ (for taste-violating terms) or $\alpha_{S} a^{2}$ (for "generic" discretization effects in the physical parameters). We then set valence and sea quark masses equal (i.e., go to "full QCD"), and iteratively interpolate in $m_{s}^{\prime}$ and extrapolate in $\hat{m}^{\prime}$ until both the kaon and the pion reach their physical masses. The extrapolations of masses and decay constants in $\hat{m}^{\prime}$ (after $m_{s}^{\prime}$ has been adjusted to $m_{s}$ ) are shown as dashed red lines in Figs. 11 and [2]

We must distinguish here between the experimental meson masses, QCD masses (no electromagnetism), and those with EM effects and 


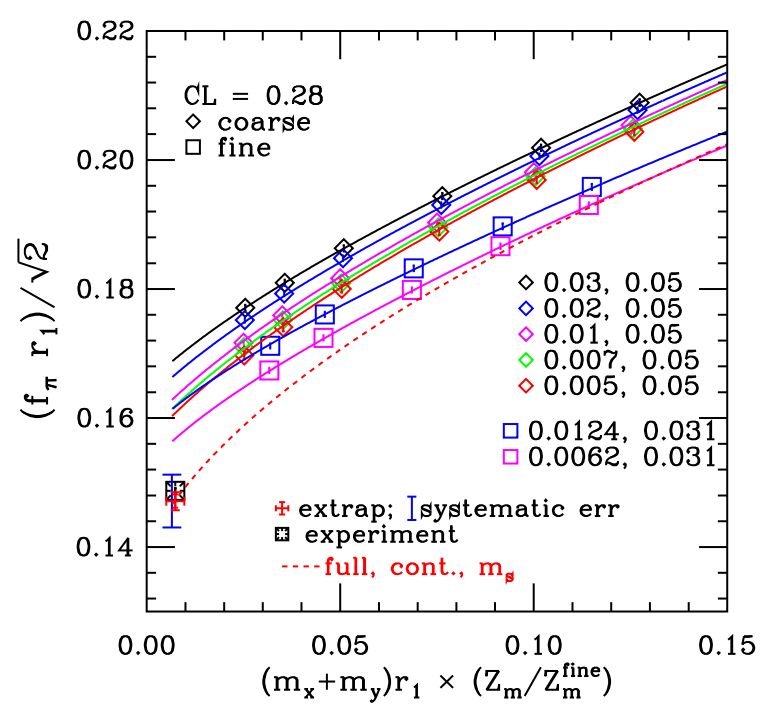

Figure 2. "Pion" decay constants with $m_{x}=m_{y}$.

isospin violations turned off, $m_{\hat{K}}$ and $m_{\hat{\pi}}$, which are the "physical" masses that determine $\hat{m}$ and $m_{s}$ in our simulation. From continuum $\chi \mathrm{PT}$, these masses are related by

$$
\begin{aligned}
m_{\hat{\pi}}^{2} & \approx\left(m_{\pi^{0}}^{\mathrm{QCD}}\right)^{2} \approx m_{\pi^{0}}^{2} \\
m_{\hat{K}}^{2} & \approx\left[\left(m_{K^{0}}^{\mathrm{QCD}}\right)^{2}+\left(m_{K^{+}}^{\mathrm{QCD}}\right)^{2}\right] / 2 \\
\left(m_{K^{0}}^{\mathrm{QCD}}\right)^{2} & \approx m_{K^{0}}^{2} \\
\left(m_{K^{+}}^{\mathrm{QCD}}\right)^{2} & \approx m_{K^{+}}^{2}-\left(1+\Delta_{E}\right)\left(m_{\pi^{+}}^{2}-m_{\pi^{0}}^{2}\right),
\end{aligned}
$$

where $m_{\pi^{0}}, m_{\pi^{+}}, m_{K^{0}}$, and $m_{K^{+}}$are the experimental masses, and $\Delta_{E}$ parametrizes the violations of Dashen's theorem 5. A conservative choice, with minimal continuum input, is $\Delta_{E}=1 \pm 1$. Using more information from the continuum gives [6] $\Delta_{E}=1.2 \pm 0.5$.

Once $\hat{m}$ and $m_{s}$ are known, we may continue the kaon extrapolation as a function of $m_{x}$ (green dashed line Fig. 11inset) until the value $m_{K^{+}}^{\mathrm{QCD}}$ is reached. This determines $m_{u}$ up to small isospinviolating corrections, which arise because $m_{u}=$ $m_{d}=\hat{m}$ for the sea quarks.

We now extrapolate the decay constants to physical quark masses, obtaining

$$
\begin{aligned}
f_{\pi} & =129.5 \pm 0.9 \pm 3.6 \\
f_{K} & =156.6 \pm 1.0 \pm 3.8 \\
f_{K} / f_{\pi} & =1.210(4)(13),
\end{aligned}
$$

where the errors are statistical and systematic (coming mainly from the chiral and continuum extrapolations, and, for dimensionful quantities, the scale error). Using Ref. [7, the result for $f_{K} / f_{\pi}$ implies $\left|V_{u s}\right|=0.2219(26)$.

For light quark masses at $2 \mathrm{GeV}$, we obtain

$$
\begin{array}{rll}
m_{u}^{\overline{\mathrm{MS}}}=1.7(0)(1)(2)(2) ; & 1.6(0)(1)(2)(1) \mathrm{MeV} \\
m_{d}^{\overline{\mathrm{MS}}}=3.9(0)(1)(4)(2) ; & 3.9(0)(1)(4)(1) \mathrm{MeV} \\
m_{u} / m_{d}=0.43(0)(1)(8) ; & 0.41(0)(1)(4),
\end{array}
$$

where the first result in each line comes from the conservative choice $\Delta_{E}=1 \pm 1$; while the second uses $\Delta_{E}=1.2 \pm 0.5[6]$. In each case the first two errors are from statistics and lattice systematics; the last, from EM effects, and the third (for $\overline{\mathrm{MS}}$ masses) is an estimate of perturbative errors 14 .

Despite the fact that we have a large number of free parameters in our fits, we believe that the chiral behavior predicted by $\mathrm{S} \chi \mathrm{PT}$ is well tested by our analysis. The evidence for this is that good fits are not possible with any of the following alternatives: (a) fits to the continuum form (36 parameters), (b) fits with all chiral logs and finite volume corrections omitted, i.e., keeping only analytic terms (38 parameters), and (c) fits with all chiral logs omitted, but finite volume corrections included (38 parameters). In subset $I I$, all these fits have $\chi^{2} /$ d.o.f. $>3$ with d.o.f. $\geq 202$.

\section{REFERENCES}

1. C. Aubin et al. (HPQCD, MILC, UKQCD), Phys. Rev. D 70 (2004) 031504(R).

2. For details, see C. Aubin et al. (MILC), hep-lat/0402030 and Ref. 4].

3. W. Lee and S. Sharpe, Phys. Rev. D60 (1999) 114503; C. Bernard, Phys. Rev. D 65 (2002) 054031; C. Aubin and C. Bernard, Phys. Rev. D68 (2003) 034014 and 074011.

4. C. Aubin et al. (MILC), hep-lat/0407028.

5. R.F. Dashen, Phys. Rev. 183 (1969) 1245.

6. We thank J. Donoghue for this summary, based on: J. Bijnens and J. Prades, Nucl. Phys. B 490 (1997) 239; J.F. Donoghue and A.F. Perez, Phys. Rev. D 55 (1997) 7075; B. Moussallam, Nucl. Phys. B 504 (1997) 381.

7. W.J. Marciano, hep-ph/0402299 